Bull. Mater. Sci., Vol. 17, No. 6, November 1994, pp. 883-891. (C) Printed in India.

\title{
Microdeformations in ferroelectrics
}

\author{
E C SUBBARAO \\ Tata Research Development and Design Centre, Pune 411050 , India
}

\begin{abstract}
Ferroelectrics undergo one or more crystallographic phase transitions; which involve lattice distortions. The direction of spontaneous polarization in ferroelectrics can be reoriented by an applied electric field (or mechanical stress). There is a spontaneous strain accompanying spontaneous polarization. Phase transitions and domain reorientations thus result in microdeformations. Many devices such as actuators and transducers are based on this behaviour. The origin of microdeformations in ferroelectrics and their consequences are discussed here.
\end{abstract}

Keywords. Microdeformations; ferroelectrics.

\section{Introduction}

It was a century ago that the first dielectric anomaly was reported, 75 years since the discovery of the first ferroelectric, namely, Rochelle-Salt, 60 years since ferroelectricity was observed in hydrogen bonded potassium dihydrogen phosphate, $\mathrm{KH}_{2} \mathrm{PO}_{4}$, and a half century since ferroelectricity was revealed in a stable oxide, namely barium titanate, $\mathrm{BaTiO}_{3}$.

The last-named family characterized by corner linking of oxygen octahedra has dominated the research and development efforts in the ferroelectric field in the last half century, because of the large number of compounds in this family, their stability and the wide range of dielectric, piezoelectric, electrostrictive and electrooptical properties exhibited by them. Oxide ferroelectrics are the subject of the present paper.

On cooling from elevated temperatures, these compounds transform from the paraelectric state to ferroelectric or antfferroelectric state at a transition temperature, called Curie temperature, $T_{\mathrm{c}}$. Some materials undergo additional phase changes at lower temperatures. Similarly, crystal symmetry may be altered by the application of electric field or mechanical stress or by compositional changes. Such structural transformations involve lattice distortions, resulting in microdeformations. The magnitude of such deformations can be modified by solid solution formation, as in the lead zirconate titanate, PZT, system. Other factors affecting microdeformations in ferroelectrics need examination.

By definition of a ferroelectric, the direction of its spontaneous polarization can be altered by an applied electric field. Such a process results in microdeformations, due to the fact that the polar axis is longer than other equivalent crystallographic directions. Domain reorientations result on application of uniaxial mechanical stress also. The magnitude of these microdeformations obviously depend upon the axial ratios and the extent of domain reorientations, and can lead to microcracking in some extreme cases. This results in a degradation of desirable dielectric and piezoelectric properties. For example, under some conditions, ferroelectric ceramics exhibit ageing phenomenon and electric fatigue, limiting their usefulness. Understanding the domain processes and the factors influencing them can be utilized to inhibit the onset of 
ageing and electric fatigue. These issues have assumed importance for demanding applications such as actuators and memory devices (e.g. DRAMs) which involve cyclic loading and unloading (electrical or mechanical) over long periods and are examined here.

\section{Phase transitions}

The most studied ferroelectrics are barium titanate and related oxygen octahedra compounds. On cooling, they undergo one or more crystallographic phase transitions starting with a paraelectric to lower-symmetry ferroelectric state. These involve lattice parameter changes and lattice distortions, which may be small as in barium titanate (Kay and Vousden 1949) (figure 1a) or large and catastrophic as in lead titanate (Shirane and Hoshino 1951) (figure 1b). These distortions are studied largely by diffraction methods (X-ray and neutron). The dimensional changes of a ceramic $\mathrm{BaTiO}_{3}$ (Shirane and Takade 1952) and $\mathrm{PbTiO}_{3}$ (Shirane and Hoshino 1951), measured by a dilatometer, are shown in figure 2 . The magnitude of ionic displacements and lattice distortions accompanying these PE-FE transitions are illustrated in figure 3 for $\mathrm{BaTiO}_{3}$ (Jona and Shirane 1962) and compared with those in $\mathrm{PbTiO}_{3}$ (Shirane et al 1956) in table 1.

The lattice parameter changes of simple perovskites (e.g. $\mathrm{BaTiO}_{3}$ and $\mathrm{PbTiO}_{3}$ ) may be compared with those of other oxygen octahedra ferroelectrics such as $\mathrm{PbNb}_{2} \mathrm{O}_{6}$

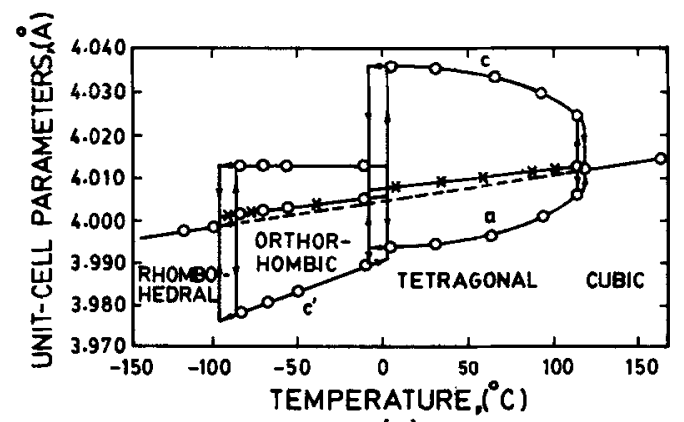

(a)

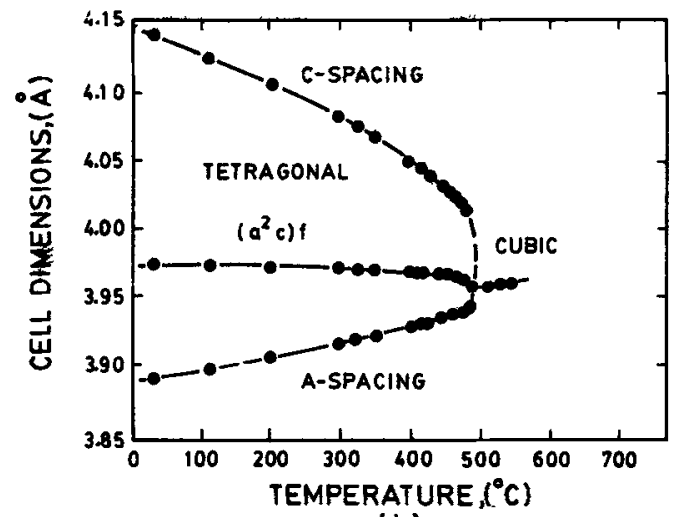

(b)

Figure 1. Temperature dependence of lattice parameters and lattice distortions of (a) $\mathrm{BaTiO}_{3}$ and (b) $\mathrm{PbTiO}_{3}$. 

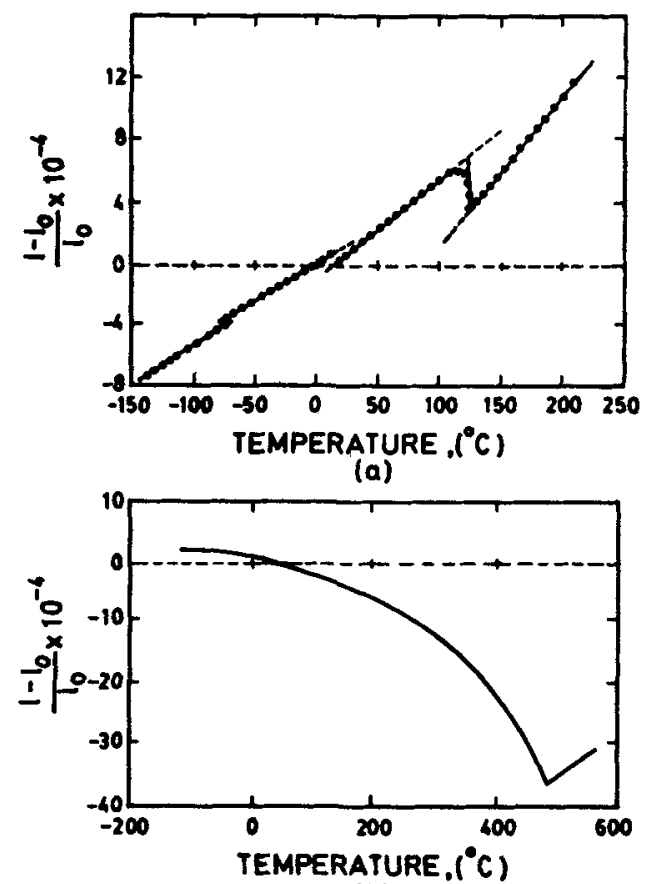

(b)

Pigure 2. Fractional length change ( $\Delta / /$ ) of ceramic (a) $\mathrm{BaTiO}_{3}$ and (b) $\mathrm{PbTiO}_{3}$.

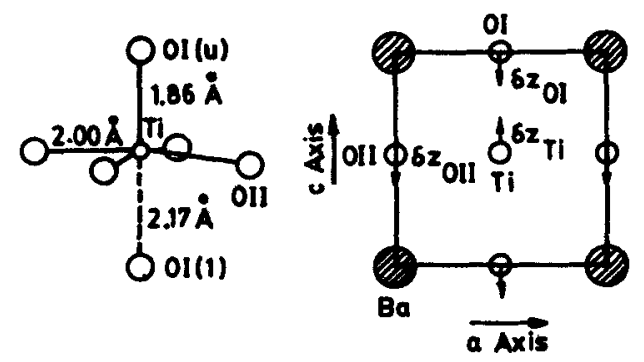

(a)

(b)

Mgure 3. Ionic displacements in tetragonal (a) $\mathrm{BaTiO}_{3}$ and (b) $\mathrm{PbTiO}_{3}$ relative to cubic phase.

Table 1. Ionic displacements (in $\mathcal{A}$ ) in $\mathrm{BaTiO}_{3}$ and $\mathrm{PbTiO}_{3}$.

\begin{tabular}{lcc}
\hline & $\mathrm{BaTiO}_{3}$ & $\mathrm{PbTiO}_{3}$ \\
\hline$\delta Z_{\mathrm{Pb}}$ or $\delta Z_{\mathrm{B}}$ & 0 & 0 \\
$\delta Z_{\mathrm{n}}$ & 0.014 & 0.17 \\
$\delta Z_{0}$ & -0.023 & 0.47 \\
$\delta Z_{\mathrm{a}}$ & -0.014 & 0.47 \\
\hline
\end{tabular}


with a tungsten bronze structure (Subbarao 1960b) (figure $4 \mathrm{a}$ ) and $\mathrm{Bi}_{4} \mathrm{Ti}_{3} \mathrm{O}_{12}$ with an Aurivillius-type layer structure (Subbarao 1961) (figure 4b). The lattice distortion at room temperature and the Curie temperature of these four compounds are listed in table 2. From these data, it is apparent that the microdeformation experienced due to lattice distortion at the phase transformation and due to anisotropic thermal contraction on further cooling to room temperature is much smaller in $\mathrm{BaTiO}_{3}, \mathrm{PbNb}_{2} \mathrm{O}_{6}$ and $\mathrm{Bi}_{4} \mathrm{Ti}_{3} \mathrm{O}_{12}$ compared to $\mathrm{PbTiO}_{3}$, which, in fact, disintegrates on cooling through the transition. This, however, has been overcome by producing $\mathrm{PbTiO}_{3}$ ceramics with a fine grain size, accomplished by the addition of about $2 \% \mathrm{Nb}$ or Ta (Subbarao 1960a). It is interesting to note that $\mathrm{Bi}_{4} \mathrm{Ti}_{3} \mathrm{O}_{12}$ (and other members of this family) have exceptionally low lattice distortion (Subbarao 1962a) in spite of their high Curie temperatures (Subbarao 1962b) (even in comparison to $\mathrm{BaTiO}_{3}$ ). The length changes experienced by ceramic specimens of $\mathrm{PbNb}_{2} \mathrm{O}_{6}$ (Subbarao 1960b) and $\mathrm{Bi}_{4} \mathrm{Ti}_{3} \mathrm{O}_{12}$ (Subbarao 1961) (figures 5a, b) relative to those of $\mathrm{BaTiO}_{3}$ and $\mathrm{PbTiO}_{3}$ (figures 2a, b) corroborate the behaviour of lattice parameter changes. These factors should have important implications for their electric fatigue behaviour on cyclic loading and unloading.

\section{Domain reorientations}

When an electric field is applied to a ferroelectric, the polar axis tends to align in the field direction. Since the polar axis is longer than equivalent crystallographic axes, the

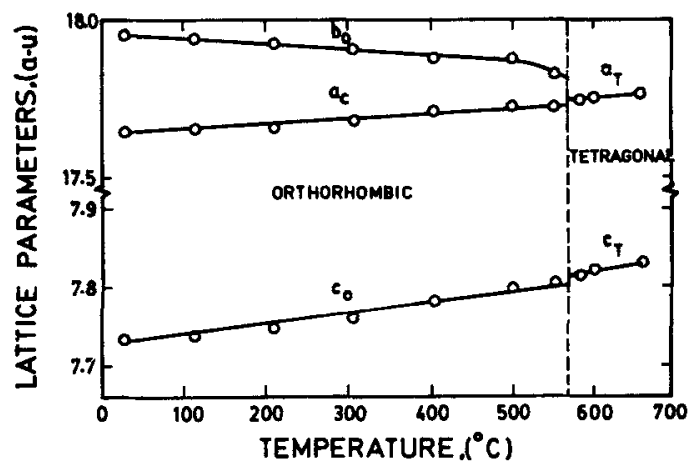

(a) of $\mathrm{PbNb}_{2} \mathrm{O}_{6}$

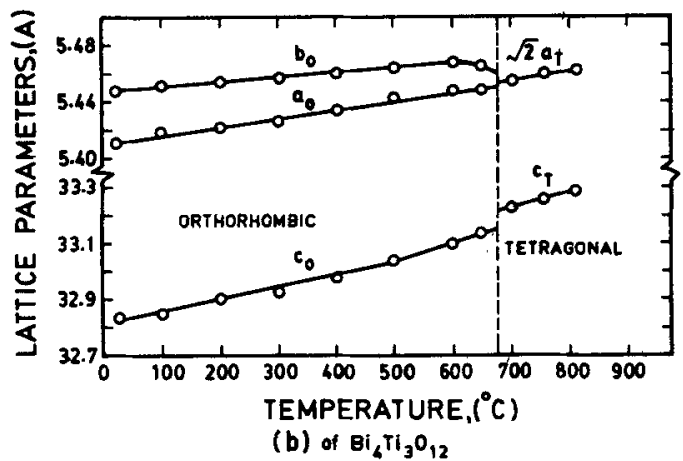

Figure 4. Lattice parameters vs temperature of (a) $\mathrm{PbNb}_{2} \mathrm{O}_{6}$ and (b) $\mathrm{Bi}_{4} \mathrm{Ti}_{3} \mathrm{O}_{12}$. 

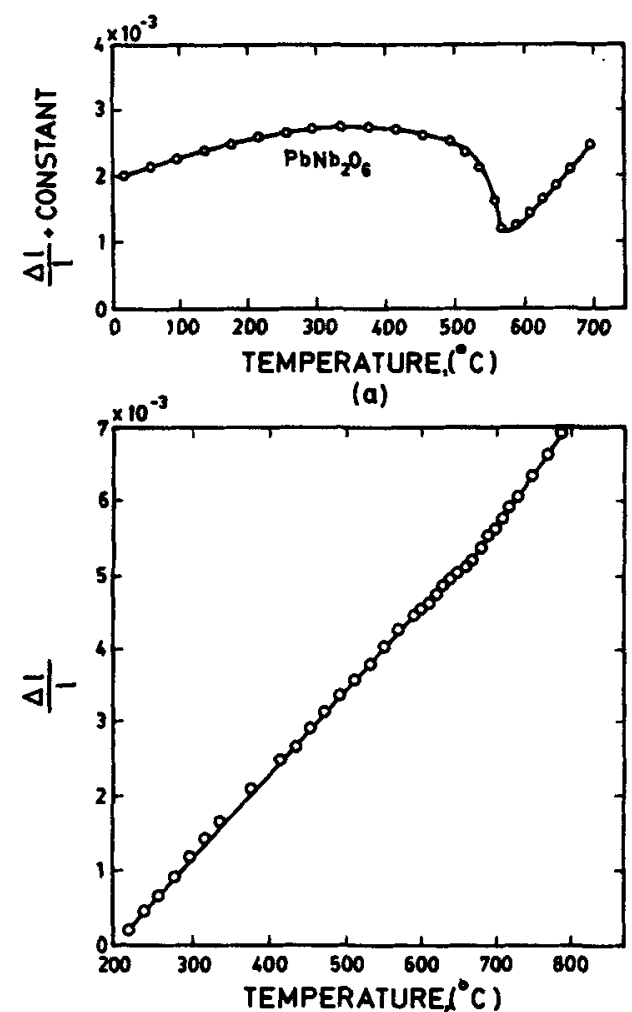

(b)

Figure 5. Fractional length change ( $\Delta / T$ ) of ceramic (a) $\mathrm{PbNb}_{2} \mathrm{O}_{6}$ and (b) $\mathrm{Bi}_{4} \mathrm{Ti}_{3} \mathrm{O}_{12}$.

Table 2. Lattice distortion at room temperature and Curie temperature $\left(T_{c}\right)$.

\begin{tabular}{lccc}
\hline & \multicolumn{2}{c}{ Lattice distortion } & \\
\cline { 2 - 3 } Compound & $b / a$ & $c / a$ & $T_{c}\left({ }^{\circ} \mathrm{C}\right)$ \\
\hline $\mathrm{BaTiO}_{3}$ & & 1.01 & 120 \\
$\mathrm{PbTiO}_{3}$ & & 1.06 & 490 \\
$\mathrm{PbNb}_{2} \mathrm{O}_{6}$ & 1.016 & & 560 \\
$\mathrm{Bi}_{4} \mathrm{Ti}_{3} \mathrm{O}_{12}$ & 1.007 & & 675 \\
\hline
\end{tabular}

process of polar or domain reorientations result in dimensional changes. The magnitude of such changes depend upon the ratio of relevant lattice parameters (e.g. $c / a$ in the case of tetragonal symmetry as in $\mathrm{BaTiO}_{3}$ and $\mathrm{PbTiO}_{3}$ and $b / a$ in the case of $\mathrm{PbNb}_{2} \mathrm{O}_{6}$ and $\mathrm{Bi}_{4} \mathrm{Ti}_{3} \mathrm{O}_{12}$ ) (table 2) and the extent of domain reorientations. The magnitude of the applied electric field as well as hindrances to domain wall motions particularly in the case of ferroelectric ceramics determine the extent of domain reorientations.

A ferroelectric ceramic which has a random orientation of polar directions to start with undergoes domain reorientations to bring about alignment of polar axis into the 
field direction when it is subjected to a d.c. electric field in a process called 'poling'. On removal of the electric field, some of the domains (imparting strain such as the 90* domains in tetragonal $\mathrm{BaTiO}_{3}$ and lead zirconate titanate, PZT) revert to their original directions (Subbarao et al 1957), leaving some degree of preferred orientation of the polar axis in the field direction. If the domain reorientation results in strain beyond the strength of the material, the sample suffers microcracking which leads to deterioration of its electrical and piezoelectric properties. Recently, acoustic emission techniques were employed to follow dimensional changes accompanying domain reorientations during poling of PZT ceramics (figure 6a) including the onset of microcracking (figure 6b) when the magnitude or duration of electric field applications is large (Subbarao et al 1993). The deterioration of electrical properties as microcracking proceeds is shown in table 3.

Similarly, acoustic emission has been used to discriminate between domain switching and microcracking of lead lanthanum zirconate titanate (PLZT) ceramics during the application of an a.c. electric field for over $10^{8}$ cycles (Jiang et al 1994).

While the above discussion emphasized the dimensional changes accompanying domain reorientations in ceramics, similar phenomena occurs in ferroelectric single crystals as well. For example, Prasad and Subbarao (1972) applied an uniaxial stress to a single crystal of $\mathrm{KNbO}_{3}$ and measured the dimensional changes in the stress direction and in the two directions perpendicular to it. An interesting relationship

$$
\Delta x / x=-\Delta y / y \text { and } \Delta z / z=0,
$$

was established and this was attributed to the reorientation of the $60^{\circ}$ domains in the orthorhombic phase of $\mathrm{KNbO}_{3}$ at room temperature. These changes were fully reversible. The duration of the application of electrical or mechanical stress on the dimensional changes has also been studied in the case of $\mathrm{BaTiO}_{3}$ (Subbarao et al 1957; Prasad and Subbarao 1973).

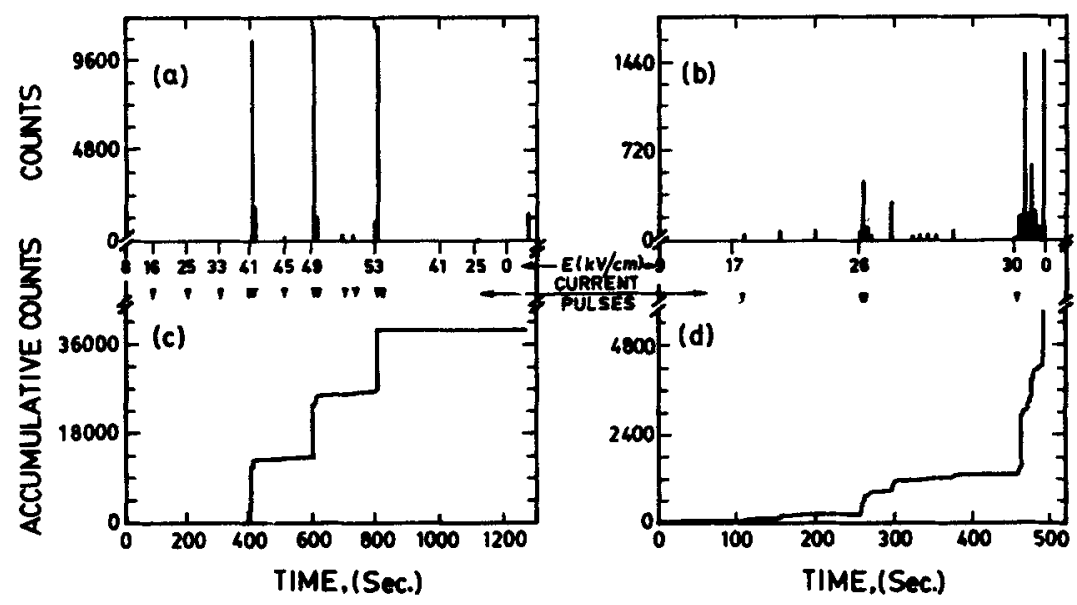

Figure 6. Acoustic emission count rate (a, c) and total AE counts (b, d) as a PZT sample was poled to successively higher fields before $(a, b)$ and after the onset $(c, d)$ of microcracking. 

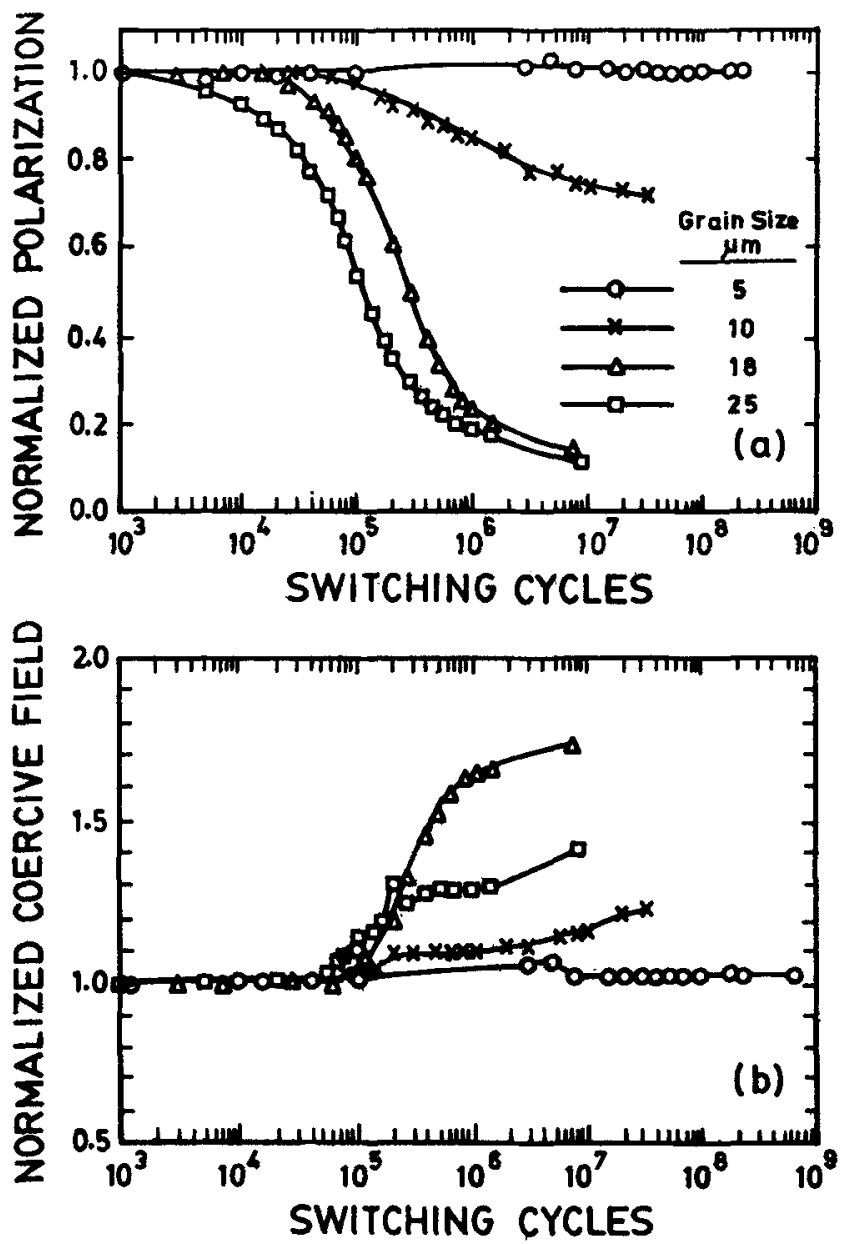

Figure 7. Normalized remanent polarization and coercive field as a function of switching cycles of hot pressed PLZT 7/68/32 ceramics with different grain sizes.

Table 3. Domain reorientations, microcracking and electrical properties of poled PZT.

\begin{tabular}{lcc}
\hline Extent of poling and microcracking & $\left(10^{-12} \mathbf{d 3}\right.$ & $\left.Q_{\mathrm{N}}\right)$ \\
\hline Poled but no onset of microcracking & 333 & 89 \\
Poled till microcracking barcly starts & 350 & 65 \\
Poled till microcracking starts & 200 & 49 \\
Poled till extensive microcracking has occurred & 119 & \\
\hline
\end{tabular}

\section{Consequences of microdeformations}

Microdeformations are a fact of life in ferroelectrics, as phase transitions and domain reorientations which take place in response to externally applied stimuli (temperature, pressure, electric field a.c. as well as d.c. and mechanical stress) inherently involve 

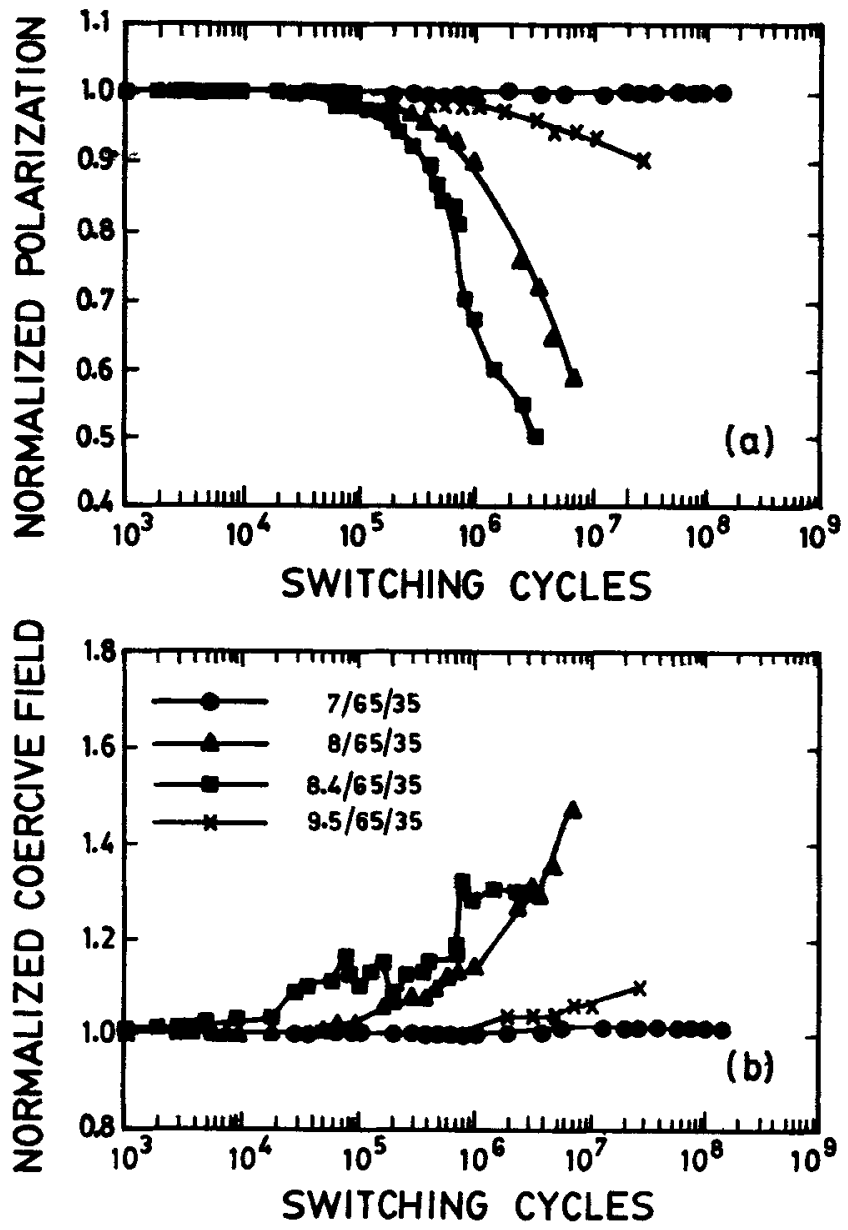

Figure 8. Normalized remanent polarization and coercive field as a function of switching cycles for hot pressed PLZT ceramics with compositions $x / 65 / 35$.

dimensional changes. Spontaneous strain accompanies spontaneous polarization in ferroelectrics.

Microdeformations indeed form the basis for many applications of ferroelectrics in piezoelectric and electrostrictive devices such as transducers and actuators. Monitoring the microdeformations during poling of piezoelectric ceramics is worthwhile in order to arrest the poling process before the onset of the microcracking of the ceramic.

For reliable long-term performance of actuators and memory devices (e.g. DRAMs) which are subjected to cyclic mechanical or electrical stress, it is necessary to identify the underlying factors which affect the domain reorientation mechanisms--both intrinsic and extrinsic. An example of an intrinsic factor is the magnitude of the lattice distortion, with a preference for a smaller value to achieve long term stability. Thus, PZT is preferred to lead titanate for transducers. Bismuth layer compounds should be good candidates for DRAMs.

Extrinsic factors which affect domain reorientations are many. As an illustration, one may cite grain size of a ceramic. The case of fine grained lead titanate is already 
mentioned (Subbarao 1960a). The PLZT ceramics suffered electric fatigue at $10^{4}$ to $10^{5}$ switching cycles when the grain size of the hot pressed ceramic is $10 \mu \mathrm{m}$ or more whereas no decay in electrical behaviour (polarization and coercive field) was detected even beyond $10^{8}$ switching cycles, if the grain size is $5 \mu \mathrm{m}$ or less (Jiang et al 1994c) (figure 7).

It is well known that cubic (paraelectric), tetragonal and rhombohedral (ferroelectric) and orthorhombic (antiferroelectric) phases can exist at room temperature in the PLZT phase diagram (Haertling 1987). Compositions near the phase boundaries can undergo phase transformations under the influence of temperature or electric field. A number of compositions near the phase boundaries in this system were studied for their electric fatigue behaviour. It is clearly established that materials with rhombohedral symmetry exhibit little or no electric fatigue compared to those with tetragonal or orthorhombic symmetry (with the degree of fatigue increasing with the lattice distortion $c / a$ or $b / a$ ) (Jiang et al 1994b). The correlation between electric fatigue and crystal symmetry was first established by selecting compositions which possess different crystal structures at room temperature (figure 8) and was later confirmed by transforming the symmetry of a given composition by changing temperature or by applying electric field. In all these cases, the rhombohedral phase with its small shear distortion exhibited the least electric fatigue.

\section{Conclusions}

Ferroelectrics which undergo crystal structure transformations and domain reorientations experience accompanying microdeformations. Many applications such as sensors and actuators depend upon the microdeformations of ferroelectrics under external stimuli. The deformations beyond certain limit can result in microcracking of ferroelectric ceramics, causing deterioration of properties. Both intrinsic and extrinsic factors affect the extent of microdeformations. An understanding of these factors and the underlying mechanisms can help in achieving reliable, long term performance of ferroelectric devices.

\section{References}

Haertling G H 1987 Ferroelectrics 7525

Jiang Q Y, Subbarao E C and Cross L E 1994a Ferroelectrics 154113

Jiang Q Y, Subbarao E C and Cross L E 1994b J. Appl. Phys. 757433

Jiang Q Y, Subbarno E C and Cross L E 1994c Acta Metall. Mater. (in press)

Jona F and Shirane G 1962 Ferroelectric crystals (New York: Macmillan Press)

Kay H F and Vousden P 1949 Philos. Mag. 401019

Prasad V C S and Subbarao E C 1972 Solid State Commun. 10811

Prasad V C S and Subbarao E C 1973 Appl. Phys. Lett. 22424

Shirane G and Hoshino S 1951 J. Phys. Soc. Jpn 6265

Shirane G and Takade A 1952 J. Phys. Soc. Jpn 71

Shirane G, Papinsky R and Frazer B C 1956 Acta Crystallogr. 9131

Subbarao E C 1960a J. Am. Ceram. Soc. 43119

Subbarao E C 1960 J. Am. Ceram. Soc. 43439

Subbarao E C 1961 Phys. Rev. 122804

Subbarao E C 1962a J. Am. Ceram. Soc. 45166

Subbarao E C 1962b J. Phys. Chem. Solids 23665

Subbarao E C. McQuarrie M C and Buessem W R 1957 J. Appl. Phys. 281194

Subbarao E C, Srikanth V, Cao W and Cross L E 1993 Ferroelectrics 145271 